\title{
Interprofessional working: a spearhead opportunity for dentistry and pharmacy
}

\author{
N. Wilson ${ }^{* 1}$ and A. Soni ${ }^{2}$
}

\section{In brief}

A challenge to dentists and pharmacists to find new ways to work together.
Encourages the integration of dentistry into general

healthcare provision.
Identifies new, patient-benefitting opportunities for dentists and pharmacists.

Dentistry and pharmacy have an opportunity, through interprofessional working, to spearhead a move towards more integrated healthcare provision, in particular for patients with chronic non-communicable diseases such as diabetes. The proposed interprofessional working poses certain challenges, but offers may opportunities and benefits.

Excellence in healthcare may be compromised when elements of care are provided in isolation.

Traditionally, dentistry has in large part been viewed, practised and regulated in a healthcare 'silo'. Posed the question: 'what do dentists and their dental teams do?' the response, even within other healthcare professions is typically: 'fix teeth', possibly qualified by 'and provide gum treatments', rather than establish and maintain oral healthcare as part of the provision of general healthcare and measures to enhance wellbeing, reflecting best evidence and state-of-the-art practice.

Faced with the substantial and growing body of evidence that oral health is important to general health and wellbeing, ${ }^{1}$ dentistry - the art and science of oral healthcare - must be more outward looking, shake off its dated image and seek opportunity to become fully integrated into the healthcare system, assuming new importance in the process. The spectre, let alone the genesis of such a metamorphosis will inevitably challenge the profession, commissioners and non-NHS providers of healthcare and, last but not least, the General Dental Council, together with other healthcare regulators, including the General Medical Council (GMC) and General Pharmaceutical Council (GPC). Indeed, to protect the public subsequent to the dawning

IImmediate-past President, British Dental Association; ${ }^{2}$ Immediate-past President of the Royal Pharmaceutical Society, Royal Pharmaceutical Society

${ }^{*}$ Correspondence to: Nairn Wilson

Email: nairn.wilson@btinternet.com

Refereed Paper. Accepted 19 October 2016

DOI: 10.1038/sj.bdj.2016.853

@British Dental Journal 2016; 221: 607-608 of a new era in integrated healthcare provision, characterised by interprofessional, holistic care, healthcare regulators may need new forward looking, harmonising legislation to allow them to develop commonality of arrangements and interlinked educational guidance, possibly in a rationalised regulatory environment. In the meantime, and despite some exemplary initiatives across the UK and internationally, including certain multinational manufacturers of oral healthcare products providing educational programmes for members of the pharmacy team, it will continue to be a sad reflection on current arrangements that oral healthcare does not typically form part of holistic care programmes developed, by way of example, for patients with chronic non-communicable diseases (CNCDs) such as diabetes.

To get from where we are to where we ought to be with interprofessional healthcare working will, in all probability, be an evolutionary process, involving behavioural change and new approaches to care planning. This process will need to be underpinned by integrated interprofessional learning and teaching at all levels, with the introduction of dental prevention and triage skills into pharmacy curricula. But where to start, and how to get traction and sustainable momentum to effect change? Setting aside the obvious first step of sign up to the vision, it is suggested that some important initial actions should be taken. For example, it is suggested that an early 'win-win' may be realised by many more dental and pharmacy teams working together at the local level.

Many individuals, in particular those with a fear of dentistry often linked to a fear of the cost of oral healthcare, seek advice from general medical practitioners and pharmacists when experiencing, among other conditions, toothache, mouth ulcers, halitosis and dentine hypersensitivity. ${ }^{2-4}$ When pharmacists are consulted they invariably do whatever they can to help such individuals, in particular those in pain. However, oral and dental considerations do not tend to be included in existing programmes of instruction in pharmacy and, in the absence of any working relationship with local dental practices, advice tends to be empirical and based on experience. As an enlightened foundation dentist commented in feedback on a lecture given recently by one of the two authors of the present paper (NW): 'I will be developing interprofessional working with local pharmacists as an associate in the practice I will be moving to, as I have found such working to be very effective and greatly appreciated by patients, in particular, patients with conditions such as diabetes.'

The concept of a close working relationship between dentists and pharmacists is not new. Notwithstanding historic links between the professions, ${ }^{5}$ in recent times dental practices have been introduced into pharmacy settings, and in some healthcare centres dental and pharmacy practices share communal space and facilities. Learning from and building on these experiences, it is suggested that the care of many patients could be transformed if dental and pharmacy practices across the UK developed, at least, effective lines of communication - possibly electronically - or, even better, were encouraged by local professional networks (LPNs) to work together to develop 
and implement innovative collaborations, contributing to 'making every contact count. From joined-up working to enhance the management of troublesome teething to collaborative ventures to improve oral health in residential care homes, with special considerations for high priority matters such smoking cessation and oral cancer screening, cross referral and other collaboration between dental and pharmacy teams could act as a catalyst to trigger other forms of interprofessional working. Furthermore, and of immediate importance, such joined-up working could, among other effects, help to increase the benefits stemming from the UK's c. $£ 1$ billion annual spend on oral hygiene aids and related products, relieve pressure on precious healthcare resources and encourage hard-to-reach individuals to become dental attenders. It could possibly even make inroads into addressing recalcitrant oral health inequalities, and contribute to further reductions in the inappropriate prescription of antibiotics in the management of, in particular, dental pain., Who knows, such interprofessional working could lead to far-reaching, funded innovations, such as dental and pharmacy teams working together with other healthcare professionals on local screening and vaccination programmes. In anticipation of the validation of the efficacy and cost efficiency of such initiatives, health regulators, educators, commissioners and other funders of dental services should be acting now to put in place arrangements to facilitate and encourage such collaborative working.

Given the above, it is suggested that dental and pharmacy teams and organisations should take early action to improve communications and devise schemes for collaborative working.
Discipline-specific, distinct approaches to healthcare provision will become increasingly difficult to defend. What better time for dentistry and pharmacy to spearhead and champion a new era in interprofessional healthcare?

1. Chapple I L C, Wilson N H F. Manifesto for a paradigm shift: periodontal health for a better life. Br Dent J 2015; 216: 159-162.

2. Dickinson C, Howlett J A, Bulman J S. The role of the community pharmacist as a dental health advisor. Comm Dent Health 1995; 12: 235-237.

3. Dickinson C, Howlett J A, Bulman J S. The community pharmacist: A dental health adviser? Pharm J 1994; 25: 262-264

4. Cope A I, Chestnut I G, Wood F, Francis N A. Dental consultations in UK general practice and antibiotic prescribing rates: a retrospective cohort study. $\mathrm{Br} J \mathrm{Gen}$ Pract 2016; 66: 329-336.

5. Anderson S. From pulling teeth to promoting oral health: pharmacy and dentistry 1815-2015. Dent Historian; in press.

6. Renton T, Wilson N H F. Understanding and managing dental and orofacial pain in general practice. $\mathrm{Br} J \mathrm{Gen}$ Pract 2016; 66: 236-237. 